\title{
Preventing Battery Attacks on Electrical Vehicles based on Data-Driven Behavior Modeling
}

\author{
Liuwang Kang \\ University of Virginia \\ Charlottesville, Virginia, 22904 \\ lk2sa@virginia.edu
}

\author{
Haiying Shen \\ University of Virginia \\ Charlottesville, Virginia, 22904 \\ hs6ms@virginia.edu
}

\begin{abstract}
With the rapid development of wireless communication technologies for electrical vehicles (EVs), as a critical part of a pure EV, batteries could be attacked (e.g., draining energy) to reduce driving range and increase driving range anxiety. However, no methods have been proposed to ensure security of $\mathrm{EV}$ batteries. In this paper, we propose the first battery attacks, which can turn on air condition and stop battery charging process by sending requests through a smartphone without being noticed by users. We then propose a Battery authentication method (Bauth) to detect the battery attacks. We firstly build a data-driven behavior model to describe a user's habits in turning on air condition and stopping battery charging. In the behavior model, to distinguish users that share a vehicle for high modeling accuracy, we apply the random forest technology to identify each user based on battery state. Based on the established behavior model, we then build a reinforcement learning model that judges whether an AC-turn-on or batter-charge-stop request from a smartphone is from the real user based on current vehicle states. We conducted real-life daily driving experiments with different participants to evaluate the battery attack detection accuracy of Bauth. The experimental results show that Bauth can prevent EV batteries from being attacked effectively in comparison with another method and its attack detection accuracy reaches as high as $93.44 \%$.
\end{abstract}

\section{CCS CONCEPTS}

- Security and privacy $\rightarrow$ Human and societal aspects of security and privacy;

\section{KEYWORDS}

Battery Attack, Data-driven Behavior Model, Reinforcement Learning, Battery Authentication

Permission to make digital or hard copies of all or part of this work for personal or classroom use is granted without fee provided that copies are not made or distributed for profit or commercial advantage and that copies bear this notice and the full citation on the first page. Copyrights for components of this work owned by others than ACM must be honored. Abstracting with credit is permitted. To copy otherwise, or republish, to post on servers or to redistribute to lists, requires prior specific permission and/or a fee. Request permissions from permissions@acm.org.

ICCPS '19, April 16-18, 2019, Montreal, QC, Canada

(C) 2019 Association for Computing Machinery.

ACM ISBN 978-1-4503-6285-6/19/04 . \$ \$15.00

https://doi.org/10.1145/3302509.3311035

\section{ACM Reference Format:}

Liuwang Kang and Haiying Shen. 2019. Preventing Battery Attacks on Electrical Vehicles based on Data-Driven Behavior Modeling. In 10th ACM/IEEE International Conference on Cyber-Physical Systems (with CPS-IoT Week 2019) (ICCPS '19), April 16-18, 2019, Montreal, QC, Canada. ACM, New York, NY, USA, 12 pages. https://doi.org/10.1145/3302509.3311035

\section{INTRODUCTION}

As a key part of pure electrical vehicles (EVs), vehicle batteries receive energy during the charging process and provide energy to drive the vehicle in the discharging process. Therefore, the driving range of a pure EV depends on the total energy stored in its batteries. Attacks on vehicle batteries can cause vehicle malfunctions and even threat to driving safety and human life. For example, an attacker may modify battery charging settings such as charging current during the charging process to generate over-high battery temperature and even battery explosion [5]. An attacker can also greatly consume battery energy without being noticed by the user to generate driving plan change and driving range anxiety. The driving range anxiety affects vehicle driving safety and may even cause driving accidents [15]. Therefore, how to improve battery security has become a challenge for car industries.

In this paper, we consider malicious adversaries that try to greatly consume battery energy or stop battery charging process without being noticed by the user in order to cause driving plan change and driving range anxiety. In practice, a malicious adversary may consume battery energy through different ways such as turning on auxiliary functions like headlights, rain wipers and air condition (AC). Here, we only consider turning on $\mathrm{AC}$ since $\mathrm{AC}$ has much larger power consumption than other auxiliary functions and is more difficult to be detected by the user. Though stopping battery charging process does not consume battery energy, it prevents the battery from further increasing its total energy.

Current EVs allow users to turn on AC or stop battery charging remotely by smartphones via wireless communication $[3,4]$. Researchers have shown how to turn on the heated seating and AC of Nissan Leaf EVs by connecting the vehicle through the Internet [2]. However, no previous works discuss the possible attacks on the vehicle batteries through the connected smartphone. Therefore, in this paper, we propose the first battery attacks which turn on AC and stop battery charging process through the connected smartphone. These attacks cannot be caught by the battery management system that only monitors battery state to ensure that the battery works safely. To the best of our knowledge, this is the first 
work to implement the two battery attacks on EVs through a smartphone in practice.

Also, there have been no methods for current EVs to detect such battery attacks to ensure battery security. To ensure battery security, one way is to improve smartphone security so that attackers cannot attack EV batteries through a smartphone. Many methods [8-11] have been proposed to prevent a smartphone from being attacked. Some methods $[9,11]$ try to remove malicious Apps from centralized mobile marketplaces. However, malware authors keep developing new methods to help attackers penetrate into marketplaces [19]. Other methods $[8,10]$ develop frameworks in the smartphone operating system to provide security protection to prevent the smartphones from being attacked. However, these methods assume that the frameworks are always trustworthy, which may not be true for advanced attacks that can compromise privileged system daemons. Further, these methods do not handle the two battery attacks we address in this paper.

To make the EVs resilient to the two battery attacks, we propose a Battery authentication method (Bauth) to detect the two battery energy attacks. It provides authentication on the AC-turn-on and battery-charge-stop requests based on a user's habits on these two actions. However, it is challenging to accurately learn a user's habits due to two reasons.

- First, a user's behaviors are greatly affected by vehicle driving environments such as vehicle indoor temperature and battery State of Charge (SOC).

- Second, the situation where more than one users share one EV exists in the household and becomes popular because of the benefits such as insurance discount, which makes it more difficult to accurately distinguish a user's habits.

To handle these challenges, we build a reinforcement learning model that can judge if a received action request from a smartphone is authentic or not.

We firstly build a data-driven behavior model to describe a user's habits in AC-turn-on and battery-charge-stop behaviors by statistically analyzing vehicle usage data. A vehicle state consists of vehicle indoor temperature and SOC. The behavior model provides vehicle state transform probability from one vehicle state to another vehicle state per time unit when AC-turn-on or battery-charge-stop action is at on or off and utilizes the state transform probability to indicate the probability that the users take different actions under certain vehicle state.

To improve the learning accuracy of the behavior model, we apply the random forest technology to identify the user based on battery state (current, battery power output and battery SOC) so that the data from different users can be classified before statistical analysis. Compared with existing classification methods, random forest based user identification method has higher identification accuracy.

And then, we use the state transform probability from the behavior model as the reward for reinforcement learning model and apply the reinforcement learning model to authorize action requests from a smartphone. More specifically, the reinforcement learning model decides to accept or reject the action request at a certain vehicle state by maximizing the

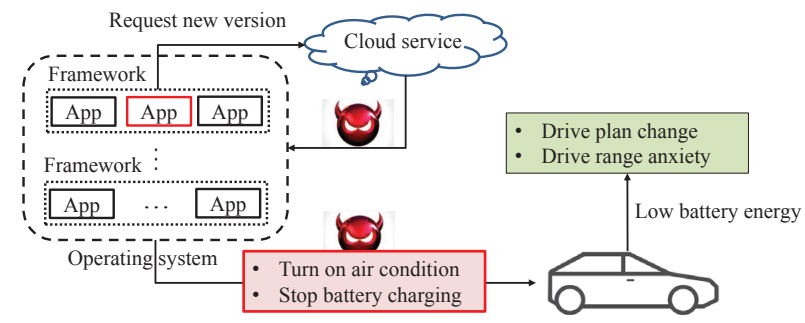

Figure 1: Battery attacks on EV batteries through a smartphone.

expected cumulative discounted reward (i.e., reflecting the user's habits on the action at the vehicle state). This way, battery attacks on EV batteries can be eliminated by Bauch to improve battery security, which helps to avoid negative effects such as driving plan change and driving range anxiety.

We firstly conduct battery attack experiments to validate the feasibility of the battery attacks through a smartphone. Then, we launched battery attacks on EVs and applied Bauth to authorize action requests from a smartphone to verify its attack detection performance in comparison with a statistical method. Our contributions are summarized below:

- We analyze the feasibility of launching the two EV battery attacks through a smartphone and how to implement them in practice, which has not been studied in previous works.

- We firstly propose a data-driven behavior model to learn a user's habits of turning on AC and stopping battery charging process based on vehicle usage data. We apply the random forest to identify users based on battery state to improve the behavior model's learning accuracy. Then, we use the behavior model to train a reinforcement learning model, which decides the action that mostly conforms to the user's actual action and thus can detect the battery attacks from a smartphone. - We conducted the first battery attack experiment to show the feasibility of such attacks on EVs. To verify the attack detection performance of Bauth, we conducted real-world experiments using total $7 \mathrm{EVs}$ in daily driving and compared attack detection results between Bauth and the statistical method. The experimental results demonstrate that the accuracy of the random forest based user identification method is around $95.70 \%$ and Bauth authorizes action requests from a smartphone with accuracy as high as $93.44 \%$.

The rest of this paper is organized as follows: Section 2 presents our proposed battery attacks on EVs. We present the details of our proposed data-driven behavior model and Bauth in Section 3 and evaluate Bauth in Section 4. Section 5 presents related research work. Finally, we conclude this paper in Section 6 with remarks on our future work.

\section{BATTERY ATTACKS ON EVS}

Our proposed battery attack remotely turns on AC or stops battery charging process through a smartphone, which is registered to connect to the EV. Figure 1 shows the details of EV battery attack process. In the smartphone operating system, vehicle mobile App and other Apps may be installed at different frameworks or just share the same framework. During the framework self-update process, code including 
battery attack is firstly downloaded from the cloud service and executed in the vehicle mobile App in the smartphone. This way, the battery attack infects the smartphone successfully and reduces EV battery energy remotely by turning on $\mathrm{AC}$ or stopping battery charging process of its connected EV.

Algorithm 1 shows pseudocode snippet in a vehicle mobile App [1] for controlling AC and battery charge process. The App firstly builds a connection between the smartphone and the EV when the user opens the vehicle mobile Ap$\mathrm{p}$ (Line 1). After successful connection, the App starts to check for possible pending requests (Line 3). When the Ap$\mathrm{p}$ receives AC-turn-on and battery-charge-stop commands from the user, it sends these requests to EV through commands climate_control and charge_control to turn on $\mathrm{AC}$ or stop battery charging process (Lines 4 and 5). Lastly, the App updates battery state and vehicle state shown in App interface with frequency of 1 time/second (Lines 6 and 7). In the battery attack, the attacker modifies parameter climate_control_instruction or charge_control_instruction to generate malicious action requests. More specifically, for a malicious battery-charge-stop request, it only happens during the battery charging process. For a malicious AC-turn-on request, it happens at any time. We divide our proposed battery attacks into No-effort attacks and Smart attacks, which are explained below.

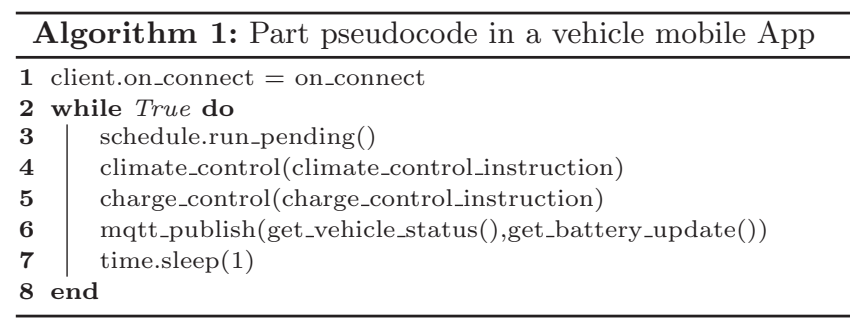

No-effort attacks No-effort attack sends malicious action requests randomly without considering whether a user is in the vehicle. Its objective is to limit the charged energy by stopping charging process or consume EV battery energy as much as possible. Therefore, No-effort attack may stop battery charging batteries during the battery charging process or turn on AC at any time. However, No-effort attack has high possibility of being detected if the user is in the vehicle.

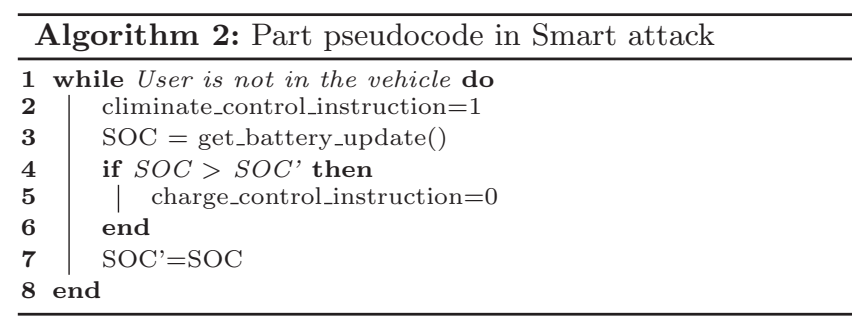

Smart attacks Here, we propose smart attacks to reduce the possibility of being detected by users. Only when the user is not in the vehicle, Smart attack will send malicious battery-charge-stop requests during the battery charging process or malicious AC-turn-on requests. In other words, Smart attack sends malicious battery-charge-stop requests

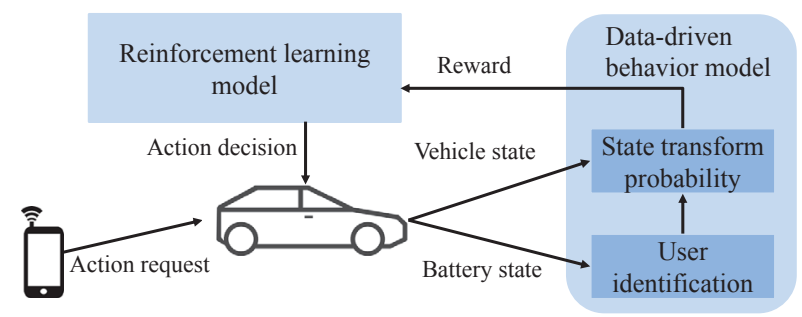

Figure 2: The architecture of Bauth for action authentication.

when the user is not in the vehicle during the battery charging process. For malicious AC-turn-on requests, Smart attack will send them only if the user is not in the vehicle and will not consider whether the vehicle is in the battery charging process. The details about how to conduct Smart attack through the vehicle mobile App are shown in Algorithm 2.

\section{SYSTEM DESIGN OF BAUTH}

To eliminate the effects of battery attacks on EV batteries and ensure battery security, we propose Bauth for EVs. Bauth runs on a micro-controller in an EV. We present the architecture of Bauth in Figure 2. Bauth consists of two major parts: data-driven behavior model and reinforcement learning model. The data-driven behavior model part firstly uses battery state to identify the user for AC-turn-on and battery-charge-stop request authentication. More specifically, it identifies the user currently driving the EV or the user that drove the EV lastly. Here, we assume the user who drove the vehicle lastly is the person to charge the vehicle. And then, for a user, the data-driven behavior model calculates state transform probability of taking an action. Here, we calculate state transform probability by analyzing historical vehicle usage data (initial vehicle state, subsequent vehicle state, and AC-turn-on/battery-charge-stop actions) and use the state transform probability as the reward in training a reinforcement learning model. In the reinforcement learning model part, for a given vehicle state, it makes decisions on accepting or rejecting an action request (turning on $\mathrm{AC}$ or stopping charging) by maximizing cumulative rewards, i.e. reflecting the user's habits on the actions for the request. When Bauth receives an action request (turning on $\mathrm{AC}$ or stopping charging), Bauth first identifies the user. Then, its reinforcement learning model for this user decides to accept or reject this request based on the current vehicle state.

\subsection{Data-driven Behavior Model}

To judge whether a received AC-turn-on or battery-chargestop request is authentic, we hope to check if current vehicle usage and battery states match the user's habits in turning on AC or stopping charging battery. However, it is difficult to learn a user's such habits. This is because a user's behaviors are affected by factors including battery SOC. Besides, the situation where more than one users share one EV exists (e.g., in the household) and becomes popular because of benefits such as insurance discount, which makes it more difficult to accurately learn a particular user's habits. If the driving 
data of the same vehicle from different users is directly used to build the behavior model, the model accuracy will be decreased greatly. Therefore, we need to identify each user first before learning the user's habits. For this purpose, vehicle mobile App in a smartphone records vehicle usage data. For each sample in vehicle usage data, it includes battery SOC, vehicle indoor temperature and status of AC-turn-on and battery-charge-stop. Both battery SOC and status of ACturn-on and battery-charge-stop can be obtained by vehicle mobile App. The vehicle indoor temperature is measured by vehicle indoor temperature sensor and its value can be sent to the smartphone through internet. This way, the behavior model can be built based on these data samples.

3.1.1 User Identification. Several researches [7, 13] use popular classification algorithms to identify users based on driving features such as accelerator, brake and steering handle speed and have advantages such as high identification accuracy. However, the data about these driving features is difficult to be obtained in practice, which limits their application. To solve this problem without compromising the advantage, we develop a user identification method to identify users based on only battery state which is much easier to be obtained.

When a user drives an EV on the road, the user's driving behaviors can be reflected by EV battery state. For example, the battery current is positive when the user accelerates the vehicle and the current magnitude will become larger when the vehicle accelerates with a larger value. Similarly, the battery current becomes negative when the user decelerates the vehicle and its magnitude will also increase as deceleration value becomes larger. Therefore, battery state can be used to describe a user's driving behaviors and identify users. The vehicle mobile App connected to EVs provides real-time battery state. By considering that the battery state can be easily obtained through vehicle mobile App, we propose a user identification method which uses random forest technology [12] to identify the user using the battery state. Random forest classifies tasks by constructing a multitude of decision trees at training time and outputting the class result, which is the mean prediction of individual trees. Compared with other classification methods such as decision tree, support vector machine, and neural network, random forest overcomes possible over-fitting issues during the training process and has more accurate classification performance for the situation where training data has unbalanced class populations. This situation happens when users sharing the same vehicle drive the vehicle with different frequencies.

In the proposed user identification method, the real-time battery state is used by the random forest technology to identify the user. More specifically, the battery state including battery current $c$, battery power output $p$, and battery $S O C$ are the inputs of random forest. By considering that battery state has different scales, we need to normalize battery state value $x$ so that these inputs can be equally treated by the random forest during the training process. The normalization process is shown as follows: $X_{i}=\frac{x_{i}-\min \left(x_{i}\right)}{\max \left(x_{i}\right)-\min \left(x_{i}\right)}$, where $x_{i}$ represents the $i^{\text {th }}$ battery state sample $[c, p, S O C]$ and $X_{i}$ is the normalized battery state value. Based on the above equation, the normalized battery state is located between 0 and 1 and sent to random forest to identify users.

Because of the dynamic changes in traffic condition and driving environment including road surface and road gradien$\mathrm{t}$, the user usually accelerates and decelerates frequently to ensure driving safety, which results in high fluctuations in battery state values. Such fluctuations affect the identification accuracy of the random forest in training. To eliminate the effects of fluctuations, we use the statistical based method, in which the mean value of the normalized battery state $X$ from time $t-\Delta$ to $t$ is considered as the statistical battery state $\bar{X}$ at time $t$. For the same normalized battery state $X$, the time period $\Delta$ determines final statistical battery state $\bar{X}$. How to determine the optimal time period $\Delta$ so that the random forest has the highest identification accuracy becomes important for our user identification method. To determine optimal time period $\Delta$, we firstly use the statistical based method to process the same training data and calculate final statistical battery states under different time periods. And then, we train the user identification model with these final statistical battery states and use the trained models to identify users. Lastly, we compare identification accuracies among these models and use the time period of the model with the highest identification accuracy as the optimal time period. Based on the normalization and statistical process, final inputs $\bar{X}$ are obtained and used to train random forest. The random forest is an ensemble of $E$ trees $T_{1}(\bar{X}), \ldots, T_{E}(\bar{X})$, where $T_{i}$ is the $i^{t h}$ decision tree and $\bar{X}$ represents statistical battery state $[\bar{c}, \bar{p}, \overline{S O C}]$ at each time. Given a set of $n$ training samples $(\bar{X}, Y)$ where $Y$ represents user ID of $\bar{X}$, each decision tree $T$ will be firstly trained until the ensemble of $E$ trees is formed. And then, the trained random forest can identify the user based on battery state during the driving process. Details of random forest training process and how to generate final output using $E$ decision trees can be found in [18].

In summary, for multi-user sharing one vehicle situations, the user can be identified online based on our user identification method and her/his corresponding AC-turn-on behavior data will be saved for the following AC-turn-on behavior analysis. For the battery-charge-stop behavior data, by considering that the user who lastly drives the vehicle at past time periods is more likely to charge batteries, we assume that the battery charging process is always conducted by the user who lastly drives the vehicle at past time periods. Under this condition, the battery-charge-stop behavior data should belong to the user who lastly drives the vehicle and will be used for battery-charge-stop behavior analysis.

3.1.2 Calculation of State Transform Probability. A user turns on AC only when (s)he feels that the vehicle's indoor temperature is out of his/her comfort range. Some users may face the balance between the temperature and driving plan because of limited battery energy. In other words, some users would turn on AC only when the battery SOC is high enough to finish their driving plans. Therefore, AC-turn-on action is determined by both vehicle indoor temperature and 
battery SOC. For the battery charging, a user charges the EV either at public charging parking lot or at private home. The user determines the battery charging time by considering remaining battery energy and his/her vehicle-use time schedule. However, the remaining battery energy is determined by vehicle usages and driving situations such as high road traffic. Therefore, constant battery-charge scheduling of charging EV batteries does not work on EV batteries in practice. Otherwise, malicious battery-charge-stop requests can be easily detected by considering whether this request follows a driver's battery-charge schedule.

Based on the above analysis, we find that both a user's AC-turn-on and battery-charge-stop behaviors are mainly affected by vehicle indoor temperature Tem and battery SOC but there are no published researches that discuss the effects of Tem and SOC on user's AC-turn-on and battery-chargestop behaviors. Our work is the first that uses Tem and $S O C$ to predict a user's AC-turn-on and battery-charge-stop behaviors. Here, we statistically analyze the dynamics of battery state and vehicle usage data to calculate statistical probabilities of taking actions under certain vehicle state (Tem and SOC). Statistical probabilities describe a user's habits in AC-turn-on and battery-charge-stop behaviors under different vehicle states. If statistical probabilities are used as reward in reinforcement learning model shown in Section 3.2 , decisions made by reinforcement learning model will follow a user's habits and helps to authorize AC-turn-on and battery-charge-stop requests.

For a given user and a given action request (AC-turn-on or battery-charge-stop), we calculate the statistical probability of taking certain actions under different vehicle states (represented by a matrix $\mathcal{B}$ ) based on historical vehicle usage data $M$. In this paper, we use $s_{t}$ to represent vehicle state $($ Tem and $S O C)$ and $d_{t}$ to represent user actions $(0$ represents reject and 1 represents accept) on the given action request. The element $B_{s_{t}, d_{t}}$ in the matrix $\mathcal{B}$ is calculated by the following equation:

$$
B_{s_{t}, d_{t}}=\sum_{\left(s^{\prime}, d^{\prime}\right) \in M} \delta_{s_{t}, s^{\prime}} \delta_{d_{t}, d^{\prime}}
$$

where $\left(s^{\prime}, d^{\prime}\right)$ is one data sample which means that the user takes action $d^{\prime}$ at vehicle state $s^{\prime} . \delta_{x, y}$ is the Kronecker delta which equals to 1 when $x$ equals to $y$, and 0 otherwise. Other elements in the matrix equals to zero. The normalization of Equation (1) is calculated as follows:

$$
\bar{B}_{s_{t}, d_{t}}=\frac{B_{s_{t}, d_{t}}}{\sum_{d_{t} \in\{0,1\}} B_{s_{t}, d_{t}}} .
$$

Elements $\bar{B}_{s_{t}, d_{t}}$ form a matrix $\overline{\mathcal{B}}$. For each row of $\overline{\mathcal{B}}$, the sum of elements $\sum_{d_{t} \in\{0,1\}} \bar{B}_{s_{t}, d_{t}}$ equals to 1 . The matrix element $\bar{B}_{s_{t}, d_{t}}$ in the normalized matrix can describe the probability that the given user takes action $d_{t}$ under vehicle state $s_{t}$ when the number of samples in vehicle usage data is large enough. Figure 3 illustrates an example to show how to calculate the probability. These vehicle state samples over time are expressed by $s_{1}, s_{2}, s_{3}, s_{4}, s_{5}$, and $s_{6}$. Note that $s_{1}$ is the same as $s_{2}$ and $s_{5}$ is the same as $s_{6}$. We firstly

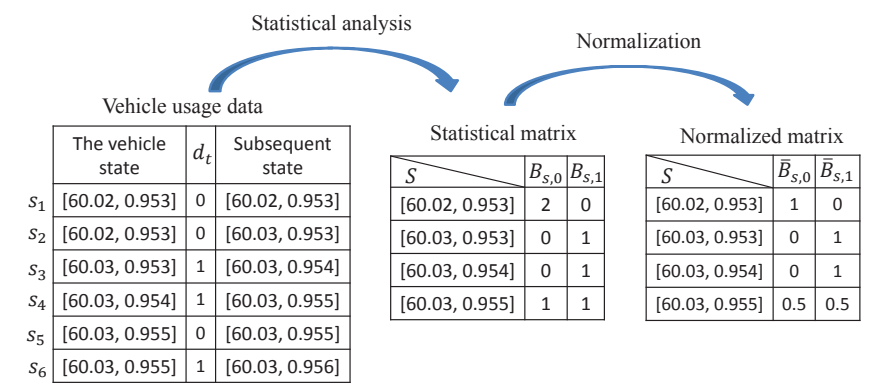

Figure 3: Statistical probability matrix calculation with vehicle usage data.

statistically analyze on the samples to calculate the number of taking action $d_{t}$ at vehicle state $s_{t}$ through Equation (1) and form the statistical matrix $\mathcal{B}$. And then, we normalize the statistical matrix to form statistical probability matrix $\overline{\mathcal{B}}$ through Equation (2).

Next, we will calculate the state transform probability based on the normalized matrix $\overline{\mathcal{B}}$. Based on the normalized matrix $\overline{\mathcal{B}}$, we calculate the normalized state transform probability matrix $\overline{\mathcal{D}}$ from one vehicle state to another vehicle state under action $d_{t} . \overline{\mathcal{D}}$ indicates how vehicle state would like to be $s_{t+1}$ if a given user takes action $d_{t}$ under $s_{t}$.

Given an initial vehicle state $s_{t}$ under action $d_{t}$, we need to calculate the normalized state transform probability from $s_{t}$ to $s_{t+1}$ (denoted by $\bar{D}_{s_{t}, d_{t}, s_{t+1}}$ ) based on the normalized element $\bar{B}_{s_{t}, d_{t}} . \bar{D}_{s_{t}, d_{t}, s_{t+1}}$ represents the probability when the user takes action $d_{t}$ and vehicle state transforms from $s_{t}$ to $s_{t+1}$. To calculate $\bar{D}_{s_{t}, d_{t}, s_{t+1}}$, we need to figure out state transform probability $D_{s_{t}, d_{t}, s_{t+1}}$ which shows the sum of state transform probabilities from $s_{t}$ to $s_{t+1}$ with action $d_{t}$. State transform probability $D_{s_{t}, d_{t}, s_{t+1}}$ is calculated as:

$$
D_{s_{t}, d_{t}, s_{t+1}}=\sum_{s^{\prime} \in S} \delta_{\left(s_{t} \rightarrow s_{t+1}\right),\left(s_{t} \rightarrow s^{\prime}\right)} \bar{B}_{s_{t}, d_{t}},
$$

where $S$ represents all possible vehicle states in vehicle usage data. Here $\left(s_{t} \rightarrow s^{\prime}\right)$ means that vehicle state changes from $s_{t}$ to $s^{\prime}$. We use $\delta_{\left(s_{t} \rightarrow s_{t+1}\right),\left(s_{t} \rightarrow s^{\prime}\right)}$ to check whether vehicle state $s^{\prime}$ changing from $s_{t}$ equals to $s_{t+1} . \delta_{\left(s_{t} \rightarrow s_{t+1}\right),\left(s_{t} \rightarrow s^{\prime}\right)}$ is 1 when $s^{\prime}$ equals to $s_{t+1}$, and 0 otherwise. We normalize state transform probability $D_{s_{t}, d_{t}, s_{t+1}}$ to obtain the normalized state transform probability $\bar{D}_{s_{t}, d_{t}, s_{t+1}}$ as follows:

$$
\bar{D}_{s_{t}, d_{t}, s_{t+1}}=\frac{D_{s_{t}, d_{t}, s_{t+1}}}{\sum_{s_{t+1} \in S} D_{s_{t}, d_{t}, s_{t+1}}} .
$$

We use the same vehicle usage data in Figure 3 to calculate state transform probability and show the calculation process in Figure 4. $s^{1}, s^{2}, s^{3}$, and $s^{4}$ represent all possible vehicle states in the sample. We firstly calculate the probability $\mathcal{D}$ of vehicle state transferring from $s_{t}$ to $s_{t+1}$ with Equation (3) based on vehicle usage data and matrix $\overline{\mathcal{B}}$ in Figure 3. And then, we normalize the state transform probability $\mathcal{D}$ to obtain the normalized state transform probability $\overline{\mathcal{D}}$.

When building the data-driven behavior model offline, Bauth runs in a micro-controller in the vehicle and collects vehicle usage data using a smartphone registered to connect the vehicle. Note that the smartphone does not need to be in 


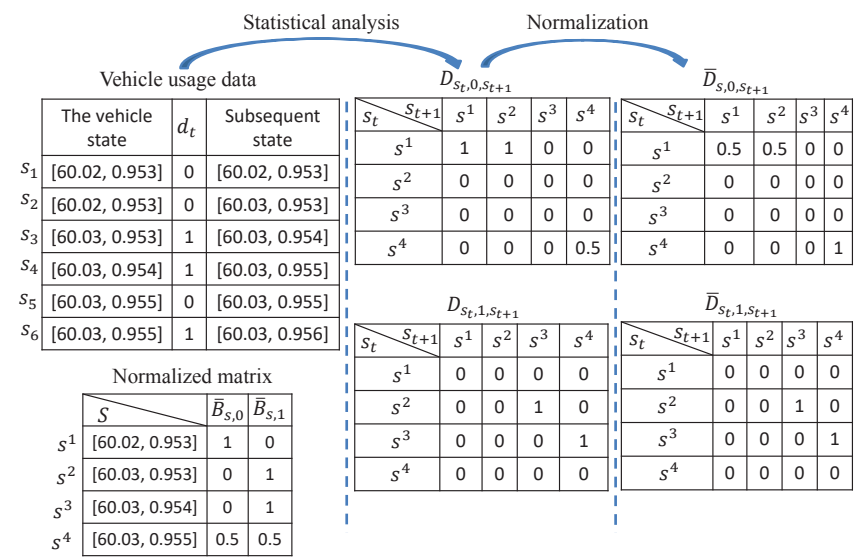

Figure 4: State transform probability matrix calculation.

the vehicle. Based on the user identification method in Section 3.1.1, Bauth identifies the user currently driving the EV (for AC-turn-on behavior learning) or the user that drove the EV lastly (for battery-charge-stop behavior learning). Then, Bauth conducts statistical analysis on the user's vehicle usage data using the methods introduced in Section 3.1.2. And then, $\bar{D}_{s_{t}, d_{t}, s_{t+1}}$ will be calculated to show the probability that the user will take action $d_{t}$ on a given action request at vehicle state $s_{t}$ and the state is transformed to $s_{t+1}$. Since the above probability can fully reflect user habits on a given action request (AC-turn-on or battery-charge-stop), we will use this probability into reinforce learning to indicate the reward when Bauth chooses decision $a_{t}$ under vehicle state $s_{t}$. That is, reward function $r\left(s_{t}, a_{t}, s_{t+1}\right)$ equals to $\bar{D}_{s_{t}, d_{t}, s_{t+1}}$, where $d_{t}$ equals to $a_{t}$, and indicates the reward after transforming from $s_{t}$ to $s_{t+1}$ with decision $a_{t}$. Here, larger reward means higher probability that the decision $a_{t}$ from Bauth will follow a user's habits. Bauth chooses decision $a_{t}$ based on total rewards it will obtain to ensure its decision $a_{t}$ follow user habits.

\subsection{Reinforcement Learning based Authentication}

Bauth uses the reinforcement learning model to authorize action requests (AC-turn-on and battery-charge-stop) from a smartphone. As shown in Figure 5, the current vehicle state is the input to the reinforcement learning model, which outputs the decision (accept or reject) on the action request. To measure the current vehicle state $s_{t}=(T e m, S O C)$, the vehicle indoor temperature sensor and vehicle mobile App firstly measure vehicle indoor temperature and battery SOC at time $t$ and send $s_{t}$ to reinforcement learning model for action authentication. Note that the vehicle mobile App in a smartphone can not only measure battery SOC but also have the function of sending action request.

In the training of the reinforcement learning model, the inputs are vehicle usage data and state transform probability matrix (considered as reward), and the output is the authentication decision $a$ from all possible options (reject or accept) for each vehicle state. The training firstly uses vehicle state

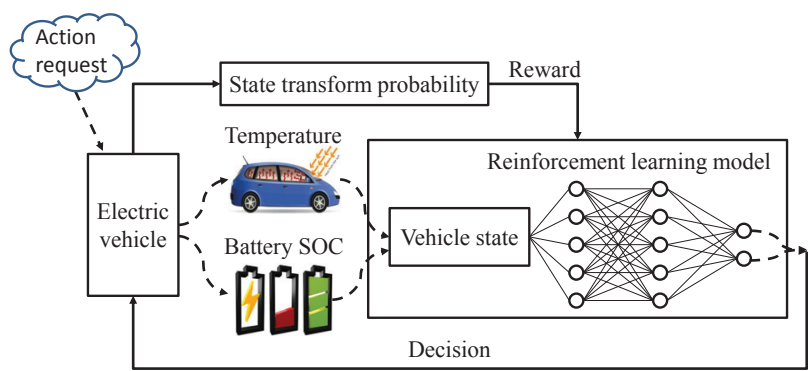

Figure 5: Reinforcement learning in Bauth.

$s_{t}$ and state transform probability matrix to calculate the expected cumulative discounted rewards $E\left[\sum_{t^{\prime}=t}^{\infty} \gamma^{\left(t^{\prime}-t\right)} r_{t^{\prime}}\right]$ (also called $Q$-value) for different authentication decisions and then outputs the authentication decision which leads to the maximum value of $E\left[\sum_{t^{\prime}=t}^{\infty} \gamma^{\left(t^{\prime}-t\right)} r_{t^{\prime}}\right]$, where $\gamma \in[0,1]$ represents discount factor, $t^{\prime}$ indicates a variable changing from $t$ to positive infinity (a value which is larger than $t$ in this paper), and $r_{t^{\prime}}$ means the reward at time $t^{\prime}$. Here, the policy is used to choose authentication decisions and calculate the expected cumulative discounted rewards. An optimal policy ensures that its authentication decisions for different vehicle states always conform actions in vehicle usage data.

3.2.1 Optimal Policy Formation. The policy $\pi$ is defined as one map $\pi: s_{t} \mapsto a_{t}$ and guides reinforcement learning model to choose authentication decision $a_{t}$ when vehicle state is $s_{t}$ in this paper. The policy $\pi$ keeps being adjusted during the training process until its authentication decisions under different vehicle states conform actions in vehicle usage data to form optimal policy $\pi^{*}$. For $\pi^{*}$, its authentication decision can follow a user's habits with high confidence. During the training process, action-value function $Q$ at initial vehicle state $s_{t}$ and authentication decision $a_{t}$ at time $t$ is introduced to indicate the expected cumulative discounted reward $E\left[\sum_{t^{\prime}=t}^{\infty} \gamma^{\left(t^{\prime}-t\right)} r_{t^{\prime}}\right]$ at vehicle state $s_{t}$ and given by:

$$
Q^{\pi}\left(s_{t}, a_{t}\right)=r\left(s_{t}, a_{t}, s_{t+1}\right)+\gamma \max Q^{\pi}\left(s_{t+1}, a_{t+1}\right) .
$$

We see that $Q^{\pi}\left(s_{t}, a_{t}\right)$ is the reward reinforcement learning model receives for entering current vehicle state $s_{t}$ plus the maximum future reward for next vehicle state $s_{t+1}$. For one vehicle state, the policy $\pi$ chooses authentication decision which can lead to the maximum value of action-value function $Q^{\pi}$. Therefore, $Q^{\pi}$ affects attack detection performance of the policy $\pi$ and needs to be calculated accurately.

$Q^{\pi}$ of the policy $\pi$ will keep being updated through vehicle usage data during the training process until the authentication decision from the policy $\pi$ maximizes its $Q$-value, and then this $\pi$ is the optimal policy $\pi^{*}$. However, it is difficult to calculate $Q$-values in action-value function and find the optimal policy through Equation (5) when the total number of all possible vehicle states is too large. For the vehicle state $s=(T e m, S O C)$, if Tem changes between $40 F$ and $90 F$ and $S O C$ changes between 0 and 1 , by considering that Tem change unit is $0.01 F$ and $S O C$ change unit is 0.001 , the total number of possible combination of different Tem and $S O C$ reaches at the $10^{6}$ level, which will result in the 
curse of dimensionality problem in practice. Besides, these many vehicle states can result in huge overload in computing $Q$-values. Therefore, rather than computing $Q$-values directly through Equation (5), we apply deep neural network into reinforcement learning model to approximate $Q$-values.

Neural networks form policy $\pi$ in reinforcement learning model and help to estimate $Q$-values under different vehicle states and choose corresponding authentication decisions. They contain two main parts: actor neural network with parameters $\theta^{\mu}$ and critic neural network with parameters $\theta^{Q}$. These parameters in the policy will be adjusted in the policy training process and the policy will better approximate $Q$-values. Section 7.1 shows how to adjust these parameters.

3.2.2 Self-Adjustment in Reward Function. For an action request from a smartphone, Bauth firstly obtains vehicle state and then generates authentication decision to prevent the battery attacks. Reward function in this paper is built by statistically analyzing historical vehicle usage data and the reward from one state to anther state in reinforcement learning model is set to state transform probability of a user's behavior. The more historical vehicle usage data is used for statistically analysis, the more accurate the reward function describes a user's habits. In practice, the historical vehicle usage samples are limited, which results in low accurate reward function. Therefore, it may result in false authentication (false alarm or missed detection) and we need to consider how to improve attack detection accuracy of Bauth.

Here, we develop a reward self-adjustment method to check whether false authentications are caused by the reward function and update the reward function based on the user action on false authentication decisions. In the reward self-adjustment model, we assume that Bauth selects false authentication decision $a_{t}^{m}$ and vehicle state changes from $s_{t}^{m}$ to $s_{t+1}^{m}$ at a false authentication situation, where $s_{t}^{m}$ and $s_{t+1}^{m}$ represent vehicle states at time $t$ and $t+1$, respectively. For this false situation, $a_{t}$ represents its correct authentication decision. The probability that Bauth selects authentication decision $a_{t}^{m}$ at vehicle state $s_{t}^{m}$ is calculated as $\bar{D}_{s_{t}^{m}, a_{t}^{m}, s_{t+1}^{m}}$. Here we use $p_{a_{t}^{m}}$ to represent $\bar{D}_{s_{t}^{m}, a_{t}^{m}, s_{t+1}^{m}}$ for simplification and $h\left(s_{t}^{m}\right)$ to represent total times of this false authentication at vehicle state $s_{t}^{m}$. The probability $p_{a_{t}}$ of selecting correct authentication decision $a_{t}$ equals to $1-p_{a_{t}^{m}}$. And then, $p_{a_{t}}$ will be compared with a threshold value $\varepsilon$ and $h\left(s_{t}^{m}\right)$ will be compared with a threshold value $\beta$ to determine whether the reward needs to be updated. $\varepsilon$ is used to indicate the minimum probability that Bauth selects authentication decision $a_{t}$ at vehicle state $s_{t}^{m} . \beta$ indicates total times of false authentication and can be set by users. If $p_{a_{t}}$ is less than $\varepsilon$ and $h\left(s_{t}^{m}\right)$ is larger than $\beta$, it means that Bauth will never select correct authentication decision and the reward of selecting correct authentication decision at this vehicle state needs to be updated to train reinforcement learning model so that reinforcement learning model can select correct authentication decision. For the new reward, $p_{a_{t}}^{\prime}$ and $p_{a_{t}^{m}}^{\prime}$ are updated to $p_{a_{t}}+\alpha\left(p_{a_{t}^{m}}-p_{a_{t}}\right)$ and $p_{a_{t}^{m}}-\alpha\left(p_{a_{t}^{m}}-p_{a_{t}}\right)$, where $\alpha$ is an update coefficient and determined based on Bauth's performance in practice. The reward in Bauth keeps being updated until authentication action from Bauth conforms action in vehicle usage data. By this way, the reward selfadjustment method helps to update the reward based on the user action on false authentication decisions and eliminate negative effects of statistical analysis on the reward function.

\subsubsection{Authentication on Action Requests from Smartphone.} The battery attack tries to attack the EV batteries by generating malicious action requests from a smartphone. For an action request from a smartphone, Bauth firstly obtain$\mathrm{s}$ vehicle state and makes its authentication decision with maximum $Q$-value. And then, Bauth sends its authentication decision to the Electronic Control Units (ECUs) in the EV. Therefore, Bauth can improve EV battery security and help eliminate negative effects of battery attacks such as driving plan change and driving range anxiety. The pseudocode of Bauth is shown in Algorithm 3.

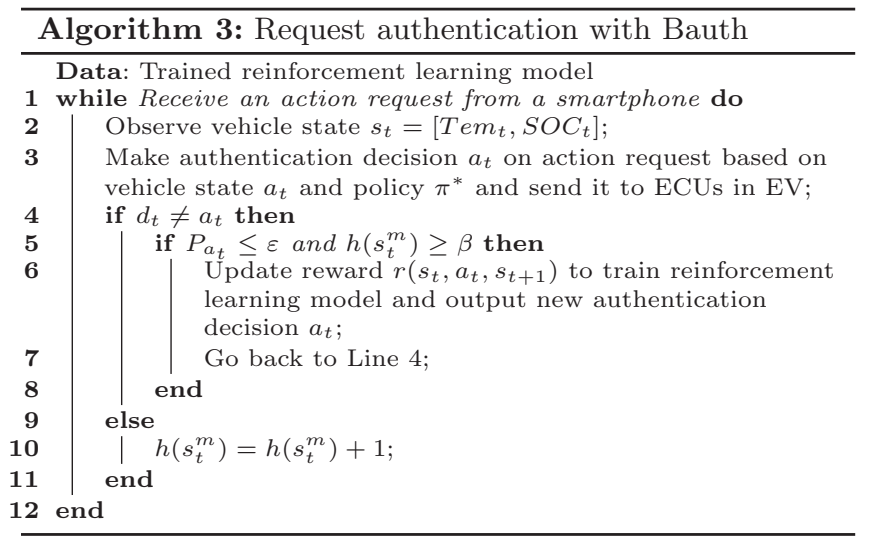

When Bauth receives an action request from a smartphone, it firstly observes vehicle state $s_{t}=\left[\mathrm{Tem}_{t}, S O C_{t}\right]$ and selects authentication decision $a_{t}$ based on vehicle state $s_{t}$ and policy $\pi^{*}$ (Line 2). And then, Bauth sends its authentication decision to ECUs in the EV to finish this action authentication process (Line 3). If the user takes action $d_{t}$ and $d_{t}$ is not the same as authentication decision $a_{t}$, total times $h\left(s_{t}^{m}\right)$ of this false authentication will increase by one. If $h\left(s_{t}^{m}\right)$ is larger than $\beta$ and probability is less than $\varepsilon$, reward $r\left(s_{t}, a_{t}, s_{t+1}\right)$ will be updated to train reinforcement learning model until new decision $a_{t}$ conforms the user action $d_{t}$ (Lines 4-11).

\section{PERFORMANCE EVALUATION}

We conducted real-world experiments based on real-life EV usage to evaluate the attack detection performance of Bauth. In the experiment, we firstly conducted EV driving experiments using total 10 participants to test identification accuracy of our user identification method. And then, we recorded AC-turn-on and battery-charge-stop behaviors of total seven EVs and utilized probability based reward function into reinforcement learning model to learn these behaviors by training reinforcement learning model with record data. Lastly, based on the established reinforcement learning model, Bauth authorized action requests from a smartphone and we compared battery attack detection results between Bauth 
and a statistical method (explained in details in Section 7.3 and abbreviated as SM) for performance evaluation.

\subsection{Experiment Settings}

We implemented battery attacks through a smartphone and run Bauth in a laptop to authorize action requests from a smartphone. To conduct battery attacks through a smartphone, we installed one App including malicious code into one Android smartphone to remotely attack EV batteries by turning on $\mathrm{AC}$ or stopping battery charging process. In the experiment malicious requests are automatically conducted using a smartphone with frequency 0.1 time/second and these requests are directly sent to both EVs and Bauth. More specifically, malicious AC-turn-on requests from No-effort attack are sent out randomly without considering whether a user is in the vehicle while malicious AC-turn-on requests from Smart attack are sent out only when the user is not in the vehicle. For malicious battery-charge-stop requests, No-effort attack and Smart attack also need to ensure that the vehicle is in the battery charging process. In EV driving experiments, total 6 male and 4 female participants (with ages range from 20 to 35 years) drove one EV on a 7.4 mile long road. During the driving process, battery state of EV batteries shown in the App was recorded with the frequency of 1 time/second to evaluate our user identification method. Details of EV driving experiments are shown in Section 7.2.

\subsection{Evaluation Aspects}

In this section, we experimentally evaluated Bauth and our experiments covered a broad set of vehicle usage cases (i.e., different numbers of users per vehicle and driving route types). Our results answer the following questions:

- How is Bauth's performance in identifying different users? Figure 6 shows identification accuracy comparisons among different users.

- How is Bauth's performance compared to other methods in terms of attack detection accuracy? Here, we define that battery attack is detected successfully in the experiment only when user or Bauth or SM detects it in 10s. Figure 7 and Figure 8 show statistical probability and state transform probability results based on given vehicle usage data, respectively. We compared attack detection results between Bauth and SM in Figure 9.

- How is the effectiveness of the self-adjustment algorithm to improve Bauth's attack detection accuracy? Figure 10 shows the effects of reward self-adjustment process on attack detection performance of Bauth.

- Is Bauth effective in different vehicle usage cases? Figure 11 shows Bauth's performances for different vehicle usage cases.

\subsection{User Identification Evaluation}

To verify the user identification method, we let total 10 participants drive one EV on a 7.4 mile long road. We utilized the K-fold cross-validation method [6] to evaluate the performance of the user identification method. K-fold crossvalidation method evaluates user identification method by partitioning the recorded battery state data into a training set (total 1350 samples) to train the user identification method and a test set (total 450 samples) to evaluate its identification accuracy. Figure 6(a) compares identification accuracies among participants. Here, identification accuracy is calculated as the rate of times when a user is identified correctly by user identification method over all samples of this user. The average value of identification accuracies among these users reaches $95.70 \%$. Besides, we compared identification performances among random forest and existing identification technologies including support vector machines (SVM), Decision Tree, and k-nearest neighbors (KNN) in Figure 6(b) when time period $\Delta$ increases gradually from $1 s$ to $60 \mathrm{~s}$ with $2 s$ increase size. We see that the random forest method has higher identification accuracy than other technologies.

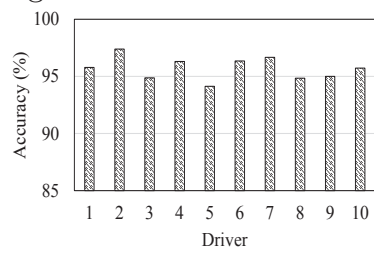

(a) Identification accuracy.

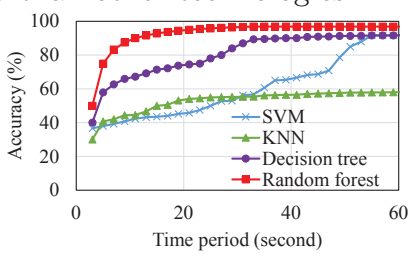

(b) Different identification methods.

Figure 6: User identification accuracy performance.

\subsection{Attack Detection Evaluation}

To evaluate attack detection performance, we applied Bauth and SM to authorize action requests from a smartphone in the last 4-day experiments, respectively. Figure 7 shows statistical probabilities of turning on $\mathrm{AC}$ and stopping charging batteries under different vehicle states for \#4 EV. Here we choose \#4 EV for performance evaluation because this EV is shared by two users and its driving route type contains Home, Office, Mall, and Supermarket, which makes \#4 EV better represent most vehicle usage cases. Based on the statistical analysis in Section 3.1.2, we calculated statistical probabilities for given vehicle state $[\mathrm{Tem}, S O C]$ and marked the corresponding probabilities with colors in Figure 7. Here, statistical probabilities equal to $\bar{B}_{s, 1}$ in Figure 3 . We see that one user would prefer turning on $\mathrm{AC}$ when vehicle indoor temperature is higher than $55 F$ and SOC is more than 0.5. For battery-charge-stop behaviors, the user often stops charging EV batteries when battery SOC is larger than 0.6. This is because users always need to ensure that battery SOC after charging process is high enough to finish their driving plan.

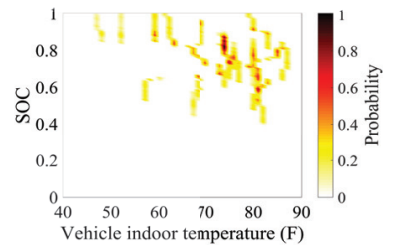

(a) AC-turn-on probability.

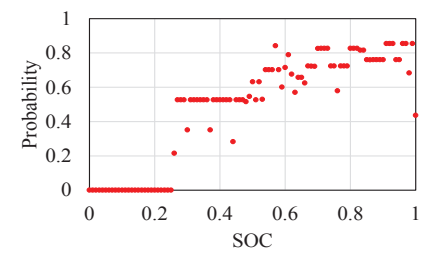

(b) Battery-charge-stop probability.
Figure 7: Statistical probabilities of AC-turn-on and battery-charge-stop.

Based on these statistical probabilities and vehicle usage data, we calculated state transform probability matrices $\overline{\mathcal{D}}$ s of turning on $\mathrm{AC}$ and stopping charging batteries and showed them in Figure 8. X-axis represents initial vehicle state and 
Y-axis represents the vehicle state after taking action (turning on AC or stopping charging batteries). Recall that state transform probability is used as reward in this paper, we see that the reward of AC-turn-on action reaches the maximum value when this action happens at around vehicle state $(72,0.8)$. For battery-charge-stop action, its reward reaches the maximum value when battery SOC is larger than 0.6. This is because the user stops charging batteries when she/he thinks battery SOC is high enough to finish driving plan.

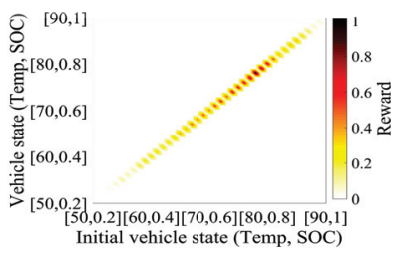

(a) Reward of AC-turn-on.

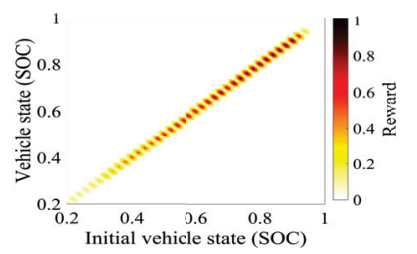

(b) Reward of battery-charge-stop.
Figure 8: The reward of AC-turn-on and batterycharge-stop for different vehicle states (vehicle indoor temperature (F) and battery SOC).

4.4.1 Performance for different attacks. To evaluate the effect$\mathrm{s}$ of training sample numbers on attack detection accuracies, we compared attack detection accuracies on No-effort attack and Smart attack for Bauth and SM under different training sample numbers of \#4 EV (changing from 1 day to 21 days) and showed attack detection accuracy results in Figure 9. Note that the situation where user may detect attacks exists in the experiment. In other words, users possibly detect battery attacks by checking a smartphone. Here we only used attack detection results of Bauth and SM for performance evaluation since attacks detected by users dominate less than $1 \%$ of all detected attacks and can be ignored for simplification. Based on Figure 9, we see that Bauth reaches higher attack detection accuracies on both No-effort attack and Smart attack compared with SM. In addition, attack detection accuracy of Bauth is more than $20 \%$ higher compared with the value of SM as total training sample numbers are more than 15 days. More specifically, if more than 15-day samples are used for training, Bauth's detection accuracy still keeps increasing while SM's attack detection accuracy will keep constant, which demonstrates Bauth's good balance between exploration and exploitation.
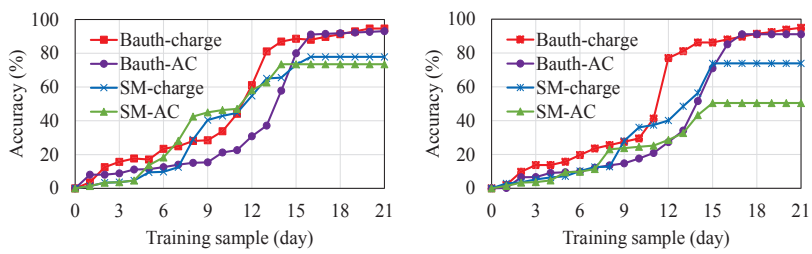

(a) Accuracy for No-effort Attacks. (b) Accuracy for Smart Attacks.

Figure 9: Attack detection accuracy comparisons for No-effort attacks and Smart attacks.

Here, we took Bauth and SM which are trained with 21-day samples as examples to better understand attack detection performance of Bauth on different battery attacks. Figure 10 shows attack detection accuracies on No-effort attack and Smart attack for Bauth and SM. For SM, its maximum attack detection accuracy is less than $80 \%$ for two different kinds of malicious action requests (AC-turn-on and battery-charge-stop). Compared with battery-chargestop requests, SM has lower attack detection accuracy on AC-turn-on requests because user decides to turn on $\mathrm{AC}$ or not by considering not only the remaining battery energy but also vehicle indoor temperature which is not considered for battery-charge-stop requests. Compared with SM, Bauth has attack detection accuracy with more than $90 \%$ on both AC-turn-on and battery-charge-stop requests. Besides, we find that SM has lower attack detection accuracies on Smart attack compared with No-effort attack, especially for ACturn-on requests. This is because Smart attack only happens when user is not in the vehicle while No-effort attack happens without considering whether user is in the vehicle, which results in more sample data to train SM for No-effort attack. For Bauth, its detection accuracy on No-effort attack is a little higher than the value on Smart attack and the average attack detection accuracy reaches 93.44\% (40.94\% higher than the value of SM), which demonstrates that Bauth has high attack detection accuracy on both No-effort attack and Smart attack. Here, Bauth has lower detection accuracy on Smart attack since Smart attack happens only when a user is not in the vehicle and Bauth detects malicious behaviors based on habits of the user who lastly drives the vehicle, which may result in attack detection errors if the user is identified wrong.

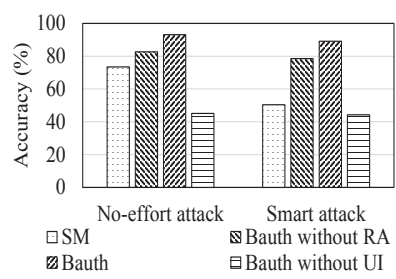

(a) AC-turn-on request.

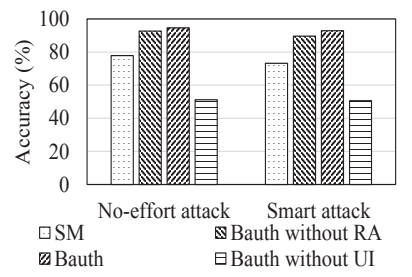

(b) Battery-charge-stop request.
Figure 10: Attack detection accuracy comparisons on AC-turn-on and battery-charge-stop requests.

Figure 10 also shows the effects of user identification (UI) and reward-adjustment (RA) on Bauth's accuracy performance. For Bauth without UI, 21-day samples of all users are directly used to form state transform probability matrix and train reinforcement learning model without identifying users. And then, reinforcement learning model makes decisions based on vehicle states. Based on Figure 10, we see that attack detection accuracies on AC-turn-on requests and battery-charge-stop requests are reduced greatly for Bauth without UI. For different users, they may share one same vehicle and usually have different habits on AC-turn-on and battery-charge-stop behaviors, which makes it hard to accurately learn habits of each user for reinforcement learning model. For Bauth without RA, it has similar training and testing processes of Bauth and the only difference is that during authentication process Bauth without RA does not adjust rewards when authentication decision is not correct. In the experiment, user recorded her/his action and sent it to 
Bauth when user's action was not the same as authentication decision. By this way, Bauth obtained user action for RA process. Figure 10 shows that attack detection accuracies on No-effort attack and Smart attack of Bauth are larger than values of Bauth without RA.

4.4.2 Performance for different vehicle usage cases. We then evaluate attack detection performance of Bauth on different vehicle usage cases. Figure 11 shows attack detection results on No-effort attack and Smart attack at different vehicle usage cases. Average attack detection accuracies for No-effort attack and Smart attack are $90.6 \%$ and $87.9 \%$ respectively, which demonstrates good authentication performance of Bauth. Bauth has the lowest attack detection accuracy at the vehicle usage case (\#3 EV) because the user does not drive \#3 EV to Office everyday and vehicle usage frequency is around 10 times/week, which had less frequency compared with other vehicles and provided limited data to train Bauth. Besides, Bauth has higher attack detection accuracy on battery-chargestop requests compared with AC-turn-on requests. This is because the user stops charging $\mathrm{EV}$ batteries or not by only considering battery SOC while the user turns on $\mathrm{AC}$ or not by considering not only vehicle indoor temperature but also battery SOC, which are more difficult to learn compared with battery-charge-stop behaviors in practice.
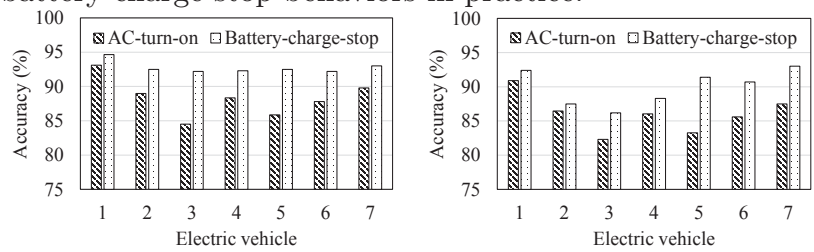

(a) Accuracy for No-effort Attacks. (b) Accuracy for Smart Attacks.

Figure 11: Bauth's attack detection accuracy performance for different vehicle usage cases.

\section{RELATED WORK}

Several existing attack defense methods $[9,11]$ have been proposed to ensure the smartphone security. Egele et al. [9] statistically analyzed mobile Apps to detect possible privacy leaks of sensitive information. Enck et al. [11] tried to understand the broader security characteristics of existing Apps by studying free Apps from the official Google Play and identify malicious Apps from centralized marketplaces. However, they are far from ideal since malware authors can find new ways to penetrate current marketplaces. Since battery attack in this paper is introduced into a smartphone during the update process of the App framework and it conducts the attack without extending mobile App permissions, it is difficult to detect battery attacks for existing attack defense methods.

Some works $[2,17]$ discuss the EV battery related security problem. Sripad et al. [17] built the battery model to analyze the potential impact of cyber-attacks utilizing the auxiliary components on the EV battery in short and long terms. Hunt [2] et al. controlled vehicle features of Nissan Leafs across the globe via the vulnerable website by knowing the vehicle identification number in advance. However, they both did not discuss the feasibility of attacks through a smartphone and how to eliminate their effects on the remaining battery energy. We design an action request authentication system to make authentication decisions on action requests from a smartphone to mitigate the effects of battery attacks.

\section{CONCLUSION}

We propose Bauth, an reinforcement learning based authentication system, to prevent EV batteries from being attacked, which helps to improve the security of EV batteries and avoid the effects of battery attacks such as driving plan change and driving range anxiety. In Bauth, we build a data-driven user behavior model to form state transform probabilities and apply them into reinforcement learning model to authorize action requests. We conduct real $\mathrm{EV}$ driving experiments to verify the performance of Bauth. The experimental results demonstrate that Bauth can detect battery attacks with high accuracy. In the future, we will improve our authentication system by considering more vehicle related variables.

\section{ACKNOWLEDGMENTS}

This research was supported in part by U.S. NSF grants NSF-1827674, CCF-1822965, OAC-1724845, ACI-1719397 and CNS-1733596, and Microsoft Research Faculty Fellowship 8300751.

\section{REFERENCES}

[1] 2016. Extract Data from Nissan Leaf API. https://github.com/ glynhudson/leaf-python-mqtt/.

[2] 2016. Nissan Leaf Electric Cars Susceptible to Hacking Attacks. https://securityzap.com/nissan/leaf/electric/cars/susceptible/ to/hacking/attacks/.

[3] 2018. NissanConnect EV. https://www.nissanusa.com/connect/ features-app/system-requirements/nissan-connect-ev/.

[4] 2018. Tesla Android and iPhone app. https://www.tesla.com/ support/android-and-iphone-app/.

[5] S Abada, G. Marlair, et al. 2016. Safety focused modeling of lithium-ion batteries: A review. J. Power Sources 306 (2016).

[6] Michael W Browne. 2000. Cross-validation methods. (2000).

[7] S. Choi, J. Kim, et al. 2007. Analysis and classification of driver behavior using in-vehicle can-bus information. In Proc. of DSP.

[8] L. Davi, A. Dmitrienko, et al. 2012. MoCFI: A Framework to Mitigate Control-Flow Attacks on Smartphones. In Proc. of NDSS.

[9] M. Egele, C. Kruegel, et al. 2011. PiOS: Detecting Privacy Leaks in iOS Applications. In Proc. of NDSS.

[10] W. Enck, P. Gilbert, S. Han, V. Tendulkar, and B. Chun. 2014. TaintDroid: an information-flow tracking system for realtime privacy monitoring on smartphones. ACM TOCS 32, 2 (2014).

[11] W. Enck, D. Octeau, and P. McDaniel. 2011. A study of android application security. In Proc. of USENIX Security Symposium.

[12] A. Liaw, M. Wiener, et al. 2002. Classification and regression by randomForest. $R$ news 2, 3 (2002).

[13] C. Miyajima, Y. Nishiwaki, et al. 2007. Driver modeling based on driving behavior and its evaluation in driver identification. Proc. of IEEE 95, 2 (2007).

[14] V. Mnih, A. Badia, et al. 2016. Asynchronous methods for deep reinforcement learning. In Proc. of ICML.

[15] N. Rauh, T. Franke, et al. 2015. Understanding the impact of electric vehicle driving experience on range anxiety. Human factors 57, 1 (2015).

[16] D. Silver, G. Lever, et al. 2014. Deterministic policy gradient algorithms. In Proc. of ICML.

[17] S. Sripad, S. Kulandaivel, et al. 2017. Vulnerabilities of Electric Vehicle Battery Packs to Cyberattacks on Auxiliary Components. arXiv preprint (2017).

[18] V. Svetnik, A. Liaw, et al. 2003. Random forest: a classification and regression tool for compound classification and QSAR modeling. j. Chem. Inf. Comput. Sci. 43, 6 (2003).

[19] C. Wu, Y. Zhou, et al. 2014. AirBag: Boosting Smartphone Resistance to Malware Infection.. In Proc. of NDSS. 


\section{APPENDIX}

\subsection{Parameter Adjustment in Reinforcement Learning Networks}

These parameters are interconnection weights in networks and will be adjusted during the policy training process to improve network performance. More specifically, both inputs of two networks are vehicle usage data and state transform probability. The outputs of actor and critic neural networks are action function $\mu\left(s_{t} \mid \theta^{\mu}\right)$ and $Q$-value $Q\left(s_{t}, a_{t} \mid \theta^{Q}\right)$ for authentication decision $a_{t}$, respectively. Here, action function $\mu\left(s_{t} \mid \theta^{\mu}\right)$ makes decisions based on $Q$-values under vehicle state $s_{t}$ and its output is authentication decision $a_{t}$ which obtains maximum $Q$-values.

Action function $\mu\left(s_{t} \mid \theta^{\mu}\right)$ specifies current policy $\pi$ by mapping vehicle state $s_{t}$ to certain authentication decision $a_{t}$ through $\operatorname{argmax}_{a_{t} \in\{0,1\}} Q\left(s_{t}, a_{t}\right)$. We utilize actor-critic algorithm [14] to adjust parameters $\theta^{Q}$ and $\theta^{\mu}$ during the policy training process so that $Q$-values are calculated accurately and authentication decision from policy $\pi$ reflects a user's habits. As one of state-of-the-art methods, the actor-critic algorithm adjusts parameters in actor neural network and critic neural network based on gradient of the expected cumulative discounted reward and approximation error, which are explained in details in the following. The key idea of actor-critic algorithm is to estimate the gradient of the expected cumulative discounted reward $E\left[\sum_{t^{\prime}=t}^{\infty} \gamma^{\left(t^{\prime}-t\right)} r_{t^{\prime}}\right]$ so that parameters in actor and critic neural networks can be adjusted. To adjust parameters $\theta^{\mu}$ in actor neural network, the gradient of the cumulative discounted reward with respect to the parameters $\theta^{\mu}$ is calculated as $\nabla_{\theta^{\mu}} E\left[\sum_{t^{\prime}=t}^{\infty} \gamma^{\left(t^{\prime}-t\right)} r_{t^{\prime}}\right]$ [16]. This gradient describes how the expected cumulative discounted reward changes when policy with parameters $\theta^{\mu}$ selects authentication decision at vehicle state $s_{t}$. Parameters $\theta^{\mu}$ will be optimized to increase the expected cumulative discounted reward by following positive gradient direction.

For critic neural network parameters $\theta^{Q}$, the difference between $Q$-values approximated by critic neural network and target $Q$-values calculated through Equation (5) is considered as the approximation error $L\left(\theta^{Q}\right)$ of critic neural network [14] and calculated as follows:

$$
L\left(\theta^{Q}\right)=E\left[\left(Q\left(s_{t}, a_{t} \mid \theta^{Q}\right)-Q^{\pi}\left(s_{t}, a_{t}\right)\right)^{2}\right],
$$

where $Q^{\pi}\left(s_{t}, a_{t}\right)$ represents $Q$-value in the policy $\pi$ and can be calculated through Equation (5). $L\left(\theta^{Q}\right)$ depends on parameters $\theta^{Q}$ in critic neural network and $\theta^{Q}$ can be updated by minimizing $L\left(\theta^{Q}\right)$ in Equation (6).

The above two paragraphes show how actor-critic algorith$\mathrm{m}$ uses the gradient and the approximation error to adjust parameters in networks. More details about how to adjust parameters in actor and critic neural networks can be found in $[14,16]$. Based on actor-critic algorithm, parameters in the policy will be adjusted in the policy training process and the policy after the training process will better approximate $Q$-values and make authentication decisions on requests. The optimal policy makes authentication decisions, which can follow a user's habits so that the battery attacks will not be authenticated.

\subsection{EV Driving Experiment Setting}

In $\mathrm{EV}$ driving experiments, the daily driving of total $7 \mathrm{EVs}$ was used for EV usage experiments and EV usage experiments lasted total 25 days long. 21-day data is used to train reinforcement learning model. 4-day data including malicious requests is used for attack detection performance evaluation. More specifically, malicious requests in a 2-day data belongs to No-effort attack and malicious requests in another 2-day data belongs to Smart attack. In the experiment, we applied Bauth and SM to authorize action requests from a smartphone in the last 4-day experiments, respectively. Besides, the user would turn off AC or restart the battery charging process if the user found attacks which were not detected successfully by Bauth and SM. In the experiment, vehicle states and AC-turn-on and battery-charge-stop behaviors were recorded through the vehicle mobile App. The vehicle indoor temperature was measured by vehicle indoor temperature sensor and its real-time measurement value was sent to the smartphone through internet. Battery state was measured and recorded by the vehicle mobile App. The vehicle usage cases such as the number of users sharing the same EV and driving route types in the EV usage experiments are shown in Table 1. Here, driving route type is determined based on locations where an EV arrives in one day. All users of these EVs charge their EVs at private homes and may have different habits in AC-turn-on and battery-charge-stop.

Table 1: Vehicle usage case

\begin{tabular}{c|c|c}
\hline EV & The number of users & Driving route type \\
\hline$\# 1$ & 1 & Home-Office \\
\hline$\# 2$ & 1 & Home-Office-Mall \\
\hline$\# 3$ & 1 & Home-Mall-Supermarket \\
\hline$\# 4$ & 2 & Home-Office-Mall-Supermarket \\
\hline$\# 5$ & 1 & Home-Office-Mall-Supermarket \\
\hline$\# 6$ & 3 &
\end{tabular}

\subsection{Statistical Method (SM) for Performance Comparison}

There exist no available methods for authorizing action requests (to turn on AC or stop battery charging process) to prevent EV batteries from battery attacks as the battery attack is firstly proposed in this paper. The most direct way is to analyze vehicle usage data to obtain statistical probabilities of taking AC-turn-on and battery-charge-stop actions under different vehicle states and utilize statistical probabilities to select decisions for action requests, which forms the statistical method (SM). For one action request at given vehicle state, SM accepts this action request if statistical probability of accepting AC-turn-on or battery-charge-stop action is higher than statistical probability of rejecting this action. Otherwise, SM rejects this action request. 


\subsection{Discussion}

Requests on heating function of air condition: In practice, AC provides not only cooling function but also heating function. In this paper, we only discuss malicious requests which try to turn on cooling function of $\mathrm{AC}$ because we did experiments at April and May at which the user only chooses cooling function. However, Bauth can also be used to authorize heating function related requests from a smartphone. Bauth can authorize heating function related requests using the same method as cooling function situations.

Effects of battery aging phenomenon: Battery ages when EV batteries experience hundreds of charging and discharging cycles. Aged batteries in EVs usually store much less energy and will affect EV performance. For example, an EV's driving range usually reduces greatly and its SOC changes much faster when its battery ages. The higher aging a battery has, the larger the battery SOC changes when EV turns on AC or stops battery charging. Therefore, previous state transform probabilities in Bauth will not accurately describe the probability of vehicle state transforming under an action. To eliminate the effects of battery aging on Bauth, we can collect vehicle usage data periodically and do statistical analysis on vehicle usage data to update state transform probabilities in Bauth. This way, state transfor$m$ probabilities can describe a user's habits accurately and Bauth detects malicious action requests with high accuracy. In this paper, we did not evaluate Bauth's performance on battery aging situations because our experiments last total 25 days long and the effects of battery aging phenomenon on $\mathrm{EV}$ performance can be ignored in practice.

Bauth implementation on EVs: In this paper, we obtain vehicle usage data and measure real-time vehicle state through vehicle mobile App in a smartphone because it is convenient to implement and participants shared their usage data to Bauth for analysis in experiments. In practice, not all users would like to send the data to Bauth. To solve this problem, we can connect CAN bus shield to On-Broad Diagnostics to read and write messages in CAN bus. The CAN bus shield reads vehicle state and action request related messages in CAN bus and sends them to Bauth. Bauth runs in a micro-controller and sends its authentication decisions to ECUs in the vehicle through CAN bus shield. Since rate powers of micro-controller and CAN bus shield are around $1 w$ and $2 w$, respectively, these rate powers are small compared with total battery power of an EV and can be ignored in practice. This way, Bauth can be implemented on EVs in practice. 